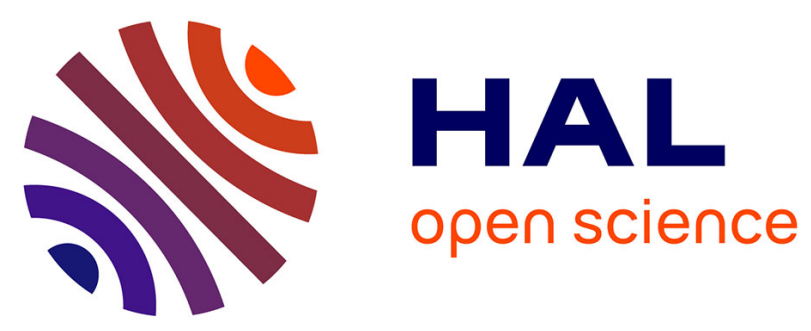

\title{
Comparison of electromagnetic transmitter and ultrasound imaging for intrafraction monitoring of prostate radiotherapy
}

Marie-Claude Biston, Timothée Zaragori, Laurent Delcoudert, Marie

Fargier-Voiron, Alexandre Munoz, Coralie Gorsse, David Sarrut, Pascal

Pommier

\section{To cite this version:}

Marie-Claude Biston, Timothée Zaragori, Laurent Delcoudert, Marie Fargier-Voiron, Alexandre Munoz, et al.. Comparison of electromagnetic transmitter and ultrasound imaging for intrafraction monitoring of prostate radiotherapy. Radiotherapy \& Oncology, 2019, 136, pp.1-8. 10.1016/j.radonc.2019.03.020 . hal-02310928

\section{HAL Id: hal-02310928 \\ https://hal.science/hal-02310928}

Submitted on 5 Oct 2020

HAL is a multi-disciplinary open access archive for the deposit and dissemination of scientific research documents, whether they are published or not. The documents may come from teaching and research institutions in France or abroad, or from public or private research centers.
L'archive ouverte pluridisciplinaire HAL, est destinée au dépôt et à la diffusion de documents scientifiques de niveau recherche, publiés ou non, émanant des établissements d'enseignement et de recherche français ou étrangers, des laboratoires publics ou privés. 


\section{Comparison of electromagnetic transmitter and ultrasound imaging for intrafraction monitoring of prostate radiotherapy}

Marie-Claude Biston ${ }^{1,2,{ }^{*}}$, Timothée Zaragori ${ }^{1, \neq}$, Laurent Delcoudert ${ }^{1, *}$, Marie Fargier-Voiron ${ }^{1}$, Alexandre Munoz $^{1}$, Coralie Gorsse ${ }^{1}$, David Sarrut ${ }^{1,2}$, Pascal Pommier ${ }^{1}$

${ }^{1}$ Léon Bérard Cancer Center, University of Lyon, F-69373 Lyon, France

${ }^{2}$ Université de Lyon, CREATIS, CNRS UMR5220, Inserm U1044, INSA, F-69622 Lyon, France

₹ These two authors have contributed equally to this work.

*Corresponding author. Department of Radiation Oncology, Centre Léon Bérard, 28 rue Laennec, 69373 Lyon Cedex 08, France

E-mail address: marie-claude.biston@lyon.unicancer.fr

Keywords: Image-Guided Radiotherapy, Prostate cancer, Ultrasounds, Electromagnetic Transmitters, monitoring 


\section{Abstract}

Background and purpose: To compare two in-beam monitoring devices for prostate radiotherapy: intraprostatic electromagnetic transmitters (EM-T) $\left(\right.$ RayPilot $^{\circledR}$, Micropos Medical) and ultrasound imaging using transperineal probe (TP-US) (Clarity ${ }^{\circledR}$, Elekta) used concomitantly on phantom and on patients.

Materials and methods: The phantom study evaluated accuracy in presence of known translations and rotations. Then intra-fraction motions were analyzed for 10 prostate cancer patients implanted with the EM-T 8 days before the simulation CT (171 sessions). The percent time in which the differences between the systems were 1 to $5 \mathrm{~mm}$ were scored for each direction.

Results: Experiments on phantom confirmed no interference between the systems and showed deviations of less than $0.5 \mathrm{~mm}$ when translations were applied progressively. In presence of rotations $\left(5-15^{\circ}\right)$, both systems displayed systematic shifts up to 6.9 and $3.8 \mathrm{~mm}$ for the TP-US and the EM-T, respectively. Absolute mean differences between displacements observed on patients with EM-T and TP-US were $\leq 0.55 \mathrm{~mm}$ in all directions except for one patient $(\leq 1.77 \mathrm{~mm})$. With an exception for this patient, a strong correlation was found in left-right direction: differences $>2 \mathrm{~mm}$ were monitored less than $0.22 \%$ of the time (mean acquisition time: 164 minutes) and never exceeded 5 seconds. Maximum differences were observed in supero-inferior direction with differences $>2 \mathrm{~mm}$ monitored more than $6.5 \%$ of the time for 3 patients. Large prostate rotations, the presence of gas and EM-T location in the prostate may explain important differences.

Conclusion: Apart from the systematic shifts induced by the rotations, the two systems were correlated and represent feasible solutions for monitoring prostate cancer treatment. 


\section{Introduction}

Hypofractionated radiotherapy protocols in prostate cancer treatment require a better accuracy in dose delivery because of an increased risk of toxicity in the surrounding tissues [1]. Critical uncertainties are expected on target localizations due to longer treatment sessions. Hence, for accurate dose delivery a robust pre-treatment imaging device combined with a real-time prostate monitoring system for correcting inter and intra-fraction motion is desirable. We studied two monitoring systems: intra-prostatic electromagnetic transmitters (EM-T) (RayPilot ${ }^{\circledR}$, Micropos Medical, Sweden) and ultrasound imaging (Clarity ${ }^{\circledR}$, Elekta, Sweden). The latter is a 2D US transperineal probe (TP-US) with robotic automated sweeping [2]. It is an alternative to other US imaging systems since it is fixed on a base plate and use the perineum as the acquisition window, which eliminates operator-dependent and quality image issues encountered with transabdominal devices [3]. Finally the probe does not interfere with the treatment beam, which enables monitoring of intrafraction motions [4]. Compared to the TP-US, EM-T is invasive [5]. A transmitter is temporarily implanted into the prostate and connected via a cable to a receptor plate positioned below the patient, on the accelerator table. The accuracy of the system was evaluated in a study performed on patients and differences of $1.7+/-1 \mathrm{~mm}$ between EM-T and 2D X-Ray images were reported [6], which makes the system reliable for intrafraction monitoring.

The objective of this study was to compare the monitoring results obtained with the two devices used concomitantly in a phantom study and on patients. Several studies dealing with monitoring devices dedicated to prostate cancer treatment can be found in the literature but none of them have been used simultaneously for treating patients mainly because of interferences between systems. The phantom study enabled to investigate the behavior of the 2 devices in presence of translations and rotations of known values. Then 171 treatments sessions of ten patients were monitored concomitantly with the two systems, enabling for the first time an in-vivo comparison.

\section{Materials and Methods}

\section{Transmitter based monitoring device}

The EM-T (RayPilot ${ }^{\circledR}$, Micropos Medical) is $3 \mathrm{~mm}$ in diameter, $17 \mathrm{~mm}$ in length and connected to a cable which is about $40 \mathrm{~cm}$ long. It is implanted in the prostate 8 days before the CT-scan by transperineal way on local or general anesthesia guided by intra-rectal US imaging. During treatment, the EM-T is connected via the cable to a receiving plate positioned on the treatment table below the patient. This plate weighting $10 \mathrm{~kg}$ is composed of a low attenuation area surrounded by two antenna arrays able to detect the EM-T shifts. Thanks to this transmitter, both translations and rotations of the target volume can be monitored in real time. The tracking frequency is $30 \mathrm{~Hz}$. After the whole treatment the transmitter is removed. 


\section{US monitoring device}

The 3D TP-US image-guided radiotherapy (IGRT) system (Clarity ${ }^{\circledR}$, Elekta, Stockholm, Sweden) is based on a 2D transperineal US probe tracked by an infrared camera [2]. For each acquisition, several hundreds of 2D US slices are acquired during an automated probe sweep performed by a step-by-step motor and combined into a 3D image. A specific immobilization device made of a base plate and two cushions for the knees enables the probe to be fixed between the patient's legs. In this work, to avoid interferences with the EM-T system, a dedicated support with no metallic component was manufactured for the US probe and directly fixed to the Raypilot $^{\circledR}$ sensor plate. During the simulation CT session, a reference US image (US ${ }_{\text {ref }}$ ) is acquired with the same patient set-up as during the CT acquisition. The US ref image is superimposed on the CT image through a calibration process, allowing the visualization of the US $\mathrm{S}_{\text {ref }}$ and CT images in the same coordinates system. A reference positioning volume (RPV) is then delineated on the US ref image. It represents the visible prostate volume on US image. Over the treatment course, a daily US image (US daily $_{\text {) }}$ is acquired at the beginning of each fraction and manually registered on the US ref image by RPV projection. The monitoring is performed via continuous 3D automatic rigid registration of the current US image with the TP-US pre-treatment image (0.7seconds per registration). Only translations are currently monitored with this system.

\section{Phantom Study}

The prostate phantom consisted in a plastic sphere with EM-T fixed on the edge. The EM-T was deliberately offcentered to reproduce the implantation as it was performed on patients (see patient data section). The sphere was attached to a water-tank support like, that allowed performing translations $(0.3 \mathrm{~mm}$ steps) and rotations (around left-right (LR) axis (pitch) and around antero-posterior (AP) axis (yaw) directions). The phantom was immersed in a parallelepiped tank full of water simulating a pelvic. The tank was positioned on the EM-T detection table. The TP-US probe was then put in contact of the tank and the EM-T connected to the table thus enabling the monitoring of the structure shifts concomitantly with the two devices (Fig.1). Several movements were studied: translations alone with regular displacements $(0.3 \mathrm{~mm}$ steps $)$; large displacements by thresholds comprised between $0.5-6 \mathrm{~mm}$; translations+rotations by $5^{\circ}$ steps, and rotations alone. Mean and standard deviations differences between the two devices were calculated for each configuration.

\section{Patient data}

Ten patients receiving a definitive irradiation of the prostate were included in this study (171 sessions) which was approved by the hospital ethics committee. Patients were implanted with the EM-T and 2 fiducial markers (FM) 8 days before the simulation CT. The EM-T was implanted in the upper base of the prostate to take into account the risk of EM-T migration during the treatment. In addition, it was also positioned laterally to limit the 
risk of injury of the urethra during the insertion process. During the simulation $\mathrm{CT}$, patients were positioned on the EM-T sensor plate placed on the CT scan table, and immobilized using the above described device for US imaging. A reference US acquisition was performed just before the CT acquisition. Patients were scanned in supine position, with $1 \mathrm{~mm}$ slice thickness and standard prostate protocol of the Brillance CT Big Bore scanner (Philips medical systems, The Netherlands).

Pre-treatment positioning was first performed with Cone Beam CT (CBCT) imaging+FM/CT registration. During CBCT imaging the monitoring modes of TP-US and EM-T were started to control patients' displacements. During treatment, irradiation was paused for shifts above a threshold of $3 \mathrm{~mm}$ for at least 15 seconds observed with both devices. If the shift lasted for more than one minute, patient positioning was adjusted based on CBCT acquisition and $\mathrm{CBCT}+\mathrm{FM} / \mathrm{CT}$ registration. For minimizing the delivered dose to the patients and limiting sessions duration, a maximum of 2 intrafraction $\mathrm{CBCT}$ per treatment session was allowed. Only one patient (patient 6) needed 2 intrafraction $\mathrm{CBCT}$ during one treatment session.

Mean prostate displacements observed with the EM-T device were first plotted against delivery time for each patient to visualize the magnitude and directions of prostate motion during treatment. Then each treatment session was analyzed to determine the difference in displacement observed between the 2 systems. Mean differences and standard deviations over the whole treatment courses were calculated on a patient-by-patient basis. The percent time differences were $>1,>1.5,>2,>2.5,>3$ and $5 \mathrm{~mm}$ was scored for each direction and each patient. To check whether differences observed were punctual or not, time thresholds of $3 s, 5 s, 10 s$ and 15 s were secondarily introduced in the analysis. The tracking time representing whole treatment sessions ranged from 90 to $200 \mathrm{~min}$. The mean tracking time was $164 \mathrm{~min}$, and the mean number of sessions was 17 (range 1220).

\section{Results}

Results of the phantom study showed that, for translations only displacements, a good agreement between TPUS and EM-T was found when progressive shifts $(0.3 \mathrm{~mm}$ steps) were carried out. Maximum differences were less than $0.4 \mathrm{~mm}$. When sudden translations varying from 0.5 to $6 \mathrm{~mm}$ were applied, larger differences were observed (Fig.2). Similarly, large punctual differences were observed because the TP-US signal was faster than EM-T to detect shifts.

When applying both translations and pitch rotations by steps of $5^{\circ}$, systematic shifts compared to reference displacement appeared for both devices (Fig.3). For the TP-US, large systematic shifts were observed in supero- 
inferior (SI) direction in presence of pitch rotation. For the EM-T device smaller systematic shifts were observed but both AP and SI directions were impacted.

When only rotations were applied the magnitudes of the systematic shifts were identical to those obtained when combining translations and rotations (Fig.4). For the TP-US, the SI direction was more impacted: pitch values of $5^{\circ}, 10^{\circ}$ and $15^{\circ}$ lead to systematic shifts of $2.5,4.9$ and $6.9 \mathrm{~mm}$, respectively. In other directions the shifts were less than $0.7 \mathrm{~mm}$. Similarly yaw rotations had low impact (maximum shifts $=1.5 \mathrm{~mm}$ for a $15^{\circ}$ rotation in $\mathrm{SI}$ direction). For the EM-T when pitch rotations were applied, shifts were comprised between 0.8-2.3mm and 1.53.8mm in AP and SI directions, respectively. Conversely to the TP-US, the EM-T device was also impacted by yaw rotations of $5-15^{\circ}$ in the 3 directions with values comprised between $1.2-4.4 \mathrm{~mm}, 1-3 \mathrm{~mm}$ and $0.5-1.8 \mathrm{~mm}$ for LR, SI and AP directions, respectively.

The relation of mean prostate displacement over all the sessions with time for each patient measured with the EM-T device is shown in Fig.5. The prostate displacement increased with longer observation time. The magnitude of the displacements was patient-dependent. They were generally more important in posterior and inferior directions. In particular, mean prostate displacements observed for patient 6 were more important than for other patients. Conversely, the frequency and magnitude of LR displacements was less important for all the patients.

The average differences between displacements measured with EM-T and TP-US are listed in Table 1 for all patients during all the treatment sessions. A good agreement was found between the 2 devices for all patients except patient 6 , with absolute mean differences being $\leq 0.55 \mathrm{~mm}$ whatever the direction. Similarly, there was a low variability ( $<0.8 \mathrm{~mm}$ except for patient 5 with $1.45 \mathrm{~mm}$ in SI direction). The best correlation was found in LR direction: mean differences were between $-0.04-0.16 \mathrm{~mm}$ and variability was $\leq 0.50 \mathrm{~mm}$. Larger deviations were found on patient 6 , particularly in SI direction where mean difference was $-1.77 \mathrm{~mm}$. A large variability was also observed in both AP and SI directions $(>3.50 \mathrm{~mm})$.

The percent time over all treatment sessions where differences between EM-T and TP-US were $>1,>1.5,>2$, $>2.5,>3$ and $5 \mathrm{~mm}$ was plotted in Fig. 6 for each direction and each patient. In LR direction, differences $>1.5 \mathrm{~mm}$ were monitored less than $2 \%$ of the time for all patients, except for patient $6(10 \%)$. Furthermore, no difference $>2 \mathrm{~mm}$ for more than $5 \mathrm{~s}$ was observed in all cases but patient 6 who had differences $>5 \mathrm{~mm}$ over a period of $15 \mathrm{~s}$ or more $1.5 \%$ of the total treatment time.

In AP direction, differences were $>1.5 \mathrm{~mm}$ less than $5 \%$ of the time for 7 patients and less than $10 \%$ of the time for 2 patients. Again for patient 6 more important deviations were found: they were $>5 \mathrm{~mm}$ more than $14 \%$ of the 
time and differences $>5 \mathrm{~mm}$ during $15 \mathrm{~s}$ were observed $13.5 \%$ of the time. Furthermore, 6 patients had no difference $>3 \mathrm{~mm}$ for more than $10 \mathrm{~s}$ and 3 patients had discordances of $3 \mathrm{~mm}$ less than $0.5 \%$ of the time.

In SI direction, deviations were less than $2 \mathrm{~mm} 99.5 \%$ of the time for 7 out of 10 patients. Two patients (number 6 and 5) had differences $>2 \mathrm{~mm}$ more than $15 \%$ of the time. They were not punctual since differences $>3 \mathrm{~mm}$ were observed for more than $15 \mathrm{~s} 9.5 \%$ and $5.3 \%$ of the time, for patient 6 and 5 respectively.

Finally, 14 intrafraction CBCT for 4 patients were performed to confirm shifts above the alert threshold (3mm for 15s) observed with the 2 monitoring devices. CT/CBCT registration results were compared to the shifts observed with the monitoring algorithm of the TP-US. Mean differences (+/SD) obtained were $0.1 \mathrm{~mm}(+/-0.12), 0.09 \mathrm{~mm}$ $(+/-0.07)$ and $0.09 \mathrm{~mm}(+/-0.09)$, in LR, AP and SI directions, respectively. Maximum difference were less than $3 \mathrm{~mm}$. Once the corrections were applied, the therapists visually controlled that both monitoring devices showed shifts below the alert threshold.

\section{Discussion}

For accurate dose delivery in prostate cancer radiotherapy, a powerful IGRT strategy for correcting interfraction motions based on soft tissue registration is required. However, recent results emphasize that the daily use of a robust interfraction repositioning device may be insufficient to obtain tumor control with reduced CTV-to-PTV margins [7-8]. Engels et al reported that, in addition with rectal distension, tight PTV margins may also be an independent prognostic factor for disease free survival in prostate cancer radiotherapy, despite the use of daily pre-treatment positioning with CBCT+FM. In addition, Loverlock et al showed that a mean of 1.7 interventions per fraction were required to keep the prostate within $2 \mathrm{~mm}$ of its planned position [8]. They also demonstrated that, without continuous monitoring of the prostate, the required posterior margin, which was set to $3 \mathrm{~mm}$, would need to be increased by $2 \mathrm{~mm}$ every 5 min starting from the time of the imaging procedure, to meet their clinical coverage requirement (PTV D95 $>90 \%$ ). These results confirm that a real-time prostate monitoring system is required for safely reducing margins. Only few modalities allowing continuous intrafraction monitoring are available: electromagnetic transponders (Calypso®) [9] or EM-T [10], ultrasound imaging and MRI-Linac [11]. MRI-Linac is a promising but expensive technology which may only concern a minority of cancer centers. We compared the results obtained with 2 recent modalities used for monitoring prostate intrafraction motion and for which few literatures are provided. The tracking algorithm accuracy of the TP-US has been recently validated on a multimodality pelvic phantom positioned on a motion platform, using an independent optical tracking system [12]. Time delay between the motion phantom and ultrasound tracking system was estimated to be $223 \pm 45$ milliseconds. They found good correlation between US-measured displacement versus optically measured displacements for both good and poor image quality (R2 $=0.958$ and 0.955 , respectively). However, the accuracy 
in presence of rotations was not evaluated and, to our knowledge, the accuracy of the monitoring algorithm was not assessed on patients.

Contrarily to Calypso $^{\circledR}$, Raypilot ${ }^{\circledR}$ is able to track with submillimeter accuracy in the 3 directions with only one implanted transmitter thanks to its length which is approximately doubled compared to Calypso ${ }^{\circledR}$ beacons. To our knowledge, there is no published study on the evaluation of the accuracy of the EM-T device on phantom and only 2 articles reporting experiences on patients. While in Kindblom et al the transmitter was inserted in the prostatic urethra [6], in Braide et al it was implanted by transperineal way, similarly to our study [10]. While their objective was to assess the pretreatment localization accuracy of the transmitter relative to marker positions, we focused on the accuracy of the monitoring process of the system.

This study is original since there is no comparative publication of similar systems on cohorts of patients mainly because the signals of electromagnetic transmitters are easily perturbed by the environment (metallic devices, electromagnetic waves). Thus, the main idea was to co-validate the monitoring algorithms of the 2 devices both in phantom study and on patients.

Experiments on phantom confirmed no interference between the systems, and showed that they were perfectly in accordance with deviations of less than $0.5 \mathrm{~mm}$, when translations were applied progressively in a given direction. Contrarily, when larger increments were applied, larger deviations were observed because of the latencies of the TP-US which was shorter than EM-T (approximate difference 10ms). When rotations were applied, both systems responded differently. Although each device compensated for the rotations with systematic shifts, these were different in terms of direction and amplitude. The TP-US showed a large systematic shift in only one direction in presence of pitch rotations while EM-T presented shifts in two directions but of lower amplitude. In addition we observed a linear relationship between the applied angle and the recorded offsets for both devices. For the TP-US, results can be explained because the algorithm used for registration of the RPV on the treatment image is only able to perform rigid translation registration. Hence rotations are compensated by translations of the RPV. Implementation of rigid registration with both translations and rotations would help increasing the accuracy of the system in presence of large rotations. Another likely explanation is that for large rotations the RPV may partly be out of the US acquisition cone which can affect the accuracy. The prostate can display large rotations during treatment $\left(>12^{\circ}\right.$ for a pitch rotation) [13]. Thus, under clinical conditions the impact of rotations on monitoring would not be negligible.

The reason of the observed systematic shifts with the EM-T device was clearly due to the transmitter that was not centered in the prostate phantom. We deliberately off-centered the transmitter in the phantom because this 
set up was more representative of our implantation process. We also noticed that the EM-T was subject to large interfractional position instability, as also observed in Braide et al [10]. Indeed, in their study they found that important transmitter shifts ( $>5 \mathrm{~mm}$ even $9 \mathrm{~mm}$ ) could happen during the treatment course, as also observed in our institution. We also did some tests with the transmitter centered and observed that applied systematic shifts were less than $2 \mathrm{~mm}$ whatever the direction even for large rotations $\left(>15^{\circ}\right)$. This raises the importance of transmitter implantation close to the center of the prostate in order to minimize the impact of rotations on the accuracy of the system.

Results on patients showed a strong correlation in 2 directions between the 2 devices, except for one patient, with maximum differences being less than $1.5 \mathrm{~mm}$ in more than $98 \%$ and $90 \%$ of the time, considering LR and AP directions, respectively. In SI direction deviations were generally more important but less than $2 \mathrm{~mm} 99.5 \%$ of the time for 7 out of 10 patients. Note that we showed on the experiment on phantom that the accuracy of both devices may be more impacted in SI direction in presence of large prostate rotations on the LR axis. When analyzing pretreatment $\mathrm{CBCT}$, we observed that patient 5 was the one having the most important prostate rotations in the LR direction over the treatment sessions (mean $-4.5^{\circ} \pm 1.70^{\circ}$ against $<1.6^{\circ}$ for other patients), which could explain the observed difference between the 2 devices in SI direction for this patient.

Results obtained on patient 6 may also be explained by prostate rotations induced by frequent gases and also because he was moving and relaxing frequently (which could also explain results in AP direction). Thus, sudden large displacements were observed and because of the difference in latency between the 2 devices, large differences over long periods could be observed.

In conclusion, apart from the systematic shifts induced by the rotations, the two systems were correlated and represent feasible solutions for monitoring prostate cancer treatment. Compared to Calypso ${ }^{\circledR}$, the EM-T device has the advantage of being removable after treatment and maybe in the future able to perform real time in vivo dosimetry. Ultrasound imaging represents a promising alternative to other modalities since it is non-invasive, non-irradiating and inexpensive. It enables visualization and registration of both prostate and organs-at-risks during the whole treatment session. However the accuracy needs to be confirmed in presence of large rotations.

\section{Acknowledgements}

This work was performed within the framework of the SIRIC LYriCAN Grant INCa-INSERM-DGOS-12563, and the LABEX PRIMES (ANR-11-LABX-0063) of Universite de Lyon, within the program Investissements d'Avenir (ANR-11-IDEX-0007) operated by the ANR. We are grateful to the Micropos ${ }^{\circledR}$ team for providing the device and transmitters. 



\section{References}

1. Clemente S, Nigro R, Oliviero C, Marchioni C, Esposito M, Giglioli FR et al. Role of the Technical Aspects of Hypofractionated Radiation Therapy Treatment of Prostate Cancer: A Review. Int J Radiat Oncol Biol Phys 2015:91(1):182-95. doi: 10.1016/j.jijobp.2014.08.006.

2. Lachaine $M$, Falco $T$. Intrafractional prostate motion management with the clarity autoscan system. Med Phys Int. 2013:1(1):72-80.

3. Fargier-Voiron M, Presles B, Pommier P, Munoz A, Rit S, Sarrut D, et al. Ultrasound versus Cone-beam CT image-guided radiotherapy for prostate and post-prostatectomy pretreatment localization. Phys Med 2015:31(8):997-1004. doi: 10.1016/j.ejmp.2015.07.147.

4. Sihono DSK, Ehmann M, Heitmann S, von Swietochowski S, Grimm M, Boda-Heggemann J, et al. Determination of Intrafraction Prostate Motion During External Beam Radiation Therapy With a Transperineal 4-Dimensional Ultrasound Real-Time Tracking System. Int J Radiat Oncol Biol Phys 2018:101(1):136-143. doi: 10.1016/j.jijobp.2018.01.040.

5. Braide K, Lindencrona U, Welinder K, Götstedt J, Ståhl I, Pettersson N, et al. Clinical feasibility and positional stability of an implanted wired transmitter in a novel electromagnetic positioning system for prostate cancer radiotherapy. Radiother Oncol 2018:128(2):336-342. doi: 10.1016/j.radonc.2018.05.031.

6. Kindblom J, Ekelund-Olvenmark AM, Syren H, lustin R, Braide K, Frank-Lissbrant I, et al. High precision transponder localization using a novel electromagnetic positioning system in patients with localized prostate cancer. Radiother Oncol 2009:90(3):307-311. doi: 10.1016/j.radonc.2018.05.031.

7. Engels B, Soete G, Gevaert T, Storme G, Michielsen D, De Ridder M. Impact of planning target volume margins and rectal distention on biochemical failure in image-guided radiotherapy of prostate cancer. Radiother Oncol 2014:111:106-109. doi: 10.1016/j.radonc.2014.02.009.

8. Lovelock DM, Messineo AP, Cox BW, Kollmeier MA, Zelefsky MJ. Continuous monitoring and intrafraction target position correction during treatment improves target coverage for patients undergoing SBRT prostate therapy. Int J Radiat Oncol Biol Phys. 2015:91(3):588-94. doi: 10.1016/j.ijrobp.2014.10.049.

9. Langen K, Willoughby T, Meeks S, Santhanam A, Cunningham A, Levine L, et al. Observations on RealTime Prostate Gland Motion Using Electromagnetic Tracking. Int J Radiat Oncol Biol Phys. 2008:15;71(4):1084-90. doi: 10.1016/j.jijrobp.2007.11.054.

10. Braide K, Lindencrona U, Welinder K, Götstedt J, Ståhl I, Pettersson N, Kindblom J. Clinical feasibility and positional stability of an implanted wired transmitter in a novel electromagnetic positioning system for prostate cancer radiotherapy. Radiother Oncol. 2018:128(2):336-342. doi: 10.1016/j.radonc.2018.05.031.

11. Kontaxis C, Bol GH, Lagendijk JJ, Raaymakers BW. A new methodology for inter- and intrafraction plan adaptation for the MR-linac. Phys Med Biol 2015:60:7485-97. doi: 10.1088/0031-9155/60/19/7485.

12. Yu AS, Najafi M, Hristov DH, Phillips T. Intrafractional Tracking Accuracy of a Transperineal Ultrasound Image Guidance System for Prostate Radiotherapy. Technol Cancer Res Treat. 2017:16(6):1067-1078. doi: $10.1177 / 1533034617728643$.

13. Amro H, Hamstra DA, Mcshan DL, Sandler H, Vineberg K, Hadley S, et al. The dosimetric impact of prostate rotations during electromagnetically guided external-beam radiation therapy. Int J Radiat Oncol Biol Phys. 2013:85(1):230-6. doi: 10.1016/j.jjrobp.2012.03.020. 


\section{Figures Legends:}

Fig1. Set-up for the phantom study. The prostate phantom consisted in a plastic sphere with EM-T fixed on the edge, immersed in a water phantom. The plastic sphere was attached to a water-tank like support. The EM-T detection plate was positioned below the phantom. The US probe was fixed on a dedicated support, with no metallic component. The probe was in contact of the water phantom.

Fig 2. Shifts recorded by the EM-T and TP-US devices when applying displacements by threshold in all directions $(A$ and $B)$, and absolute differences observed between the devices $(C$ and $D)$. Positive values are inferior, anterior and left directions.

Fig 3. Shifts recorded by the EM-T and TP-US devices when applying rotations along LR axis and displacement toward anterior direction only. Positive values are inferior, anterior and left directions.

Fig 4. Systematic shifts recorded by the EM-T and TP-US devices in LR, SI and AP directions when applying rotations only along LR (Pitch) and SI (yaw) axis.

Fig 5. Average (+/- standard deviation) prostate displacement observed in LR, SI and AP directions over all the sessions in function of the treatment session time for each patient measured with the EM-T device. Positive values are left, superior and anterior displacements.

Fig 6. Percent time over all treatment sessions where difference between EM-T and TP-US exceeds specific threshold $(\mathrm{mm})$.

Table 1. Average differences between displacements measured with EM-T and TP-US

\begin{tabular}{cccc}
\hline & \multicolumn{3}{c}{ Average difference $( \pm$ SD) } \\
\hline PR $(\mathrm{mm})$ & SI $(\mathrm{mm})$ & AP $(\mathrm{mm})$ \\
\hline Patient 1 & $0.16(0.29)$ & $-0.11(0.54)$ & $-0,02(0,69)$ \\
Patient 3 & $0.09(0.38)$ & $0.55(0.77)$ & $0.13(0.69)$ \\
Patient 4 & $0.05(0.43)$ & $0.09(0.56)$ & $0.04(0.69)$ \\
Patient 5 & $0.01(0.50)$ & $-0.03(0.33)$ & $0.05(0.62)$ \\
Patient 6 & $-0.04(0.33)$ & $-0.09(1.45)$ & $0.24(0.74)$ \\
Patient 7 & $0.41(1.41)$ & $-1.77(5.73)$ & $1.24(3.51)$ \\
Patient 8 & $0.11(0.42)$ & $-0.53(0.50)$ & $0.30(0.43)$ \\
Patient 9 & $0(0.42)$ & $-0.19(0.34)$ & $0.17(0.45)$ \\
Patient 10 & $0.02(0.25)$ & $-0.10(0.34)$ & $-0.33(0.75)$ \\
\hline
\end{tabular}




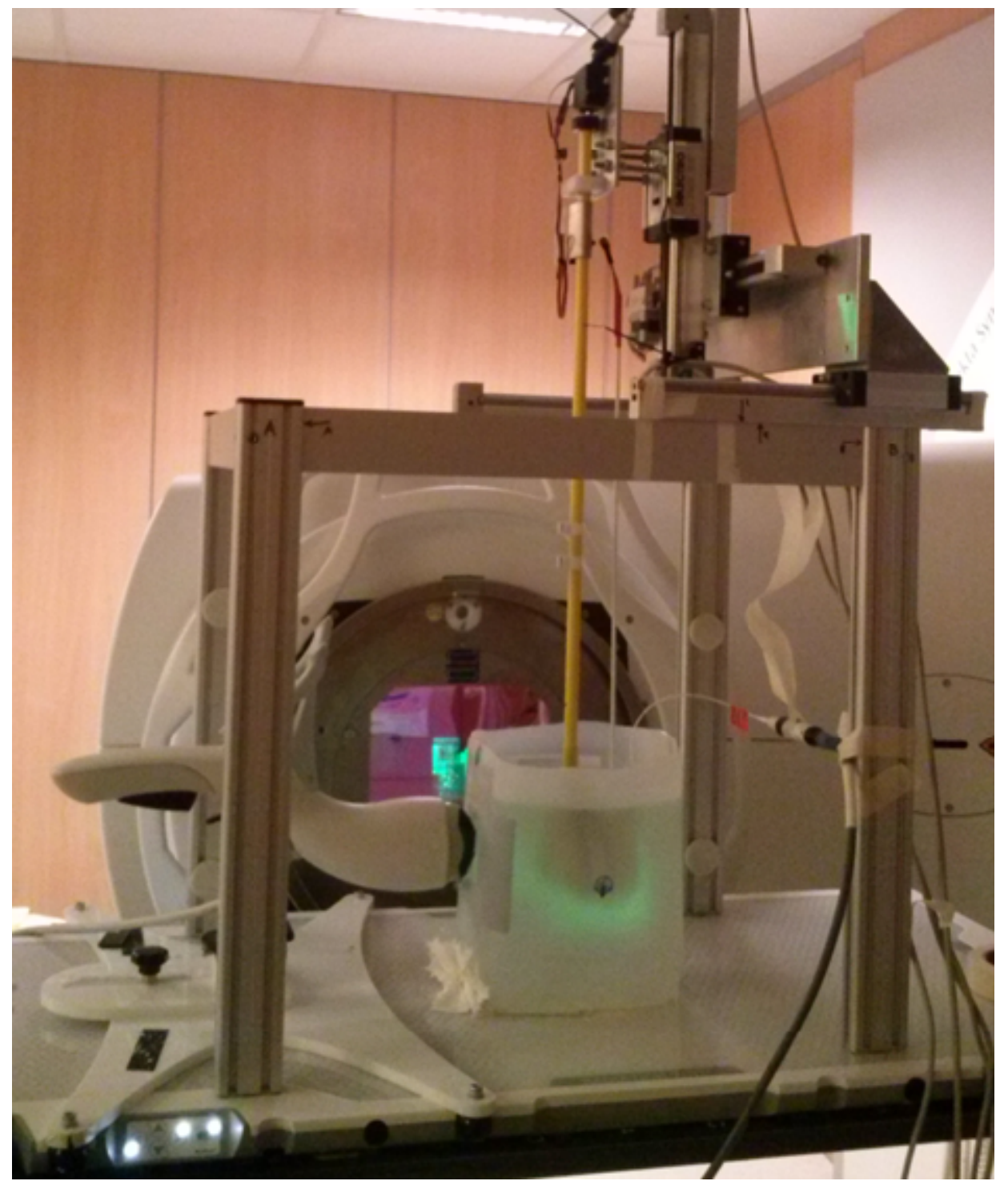


LR direction

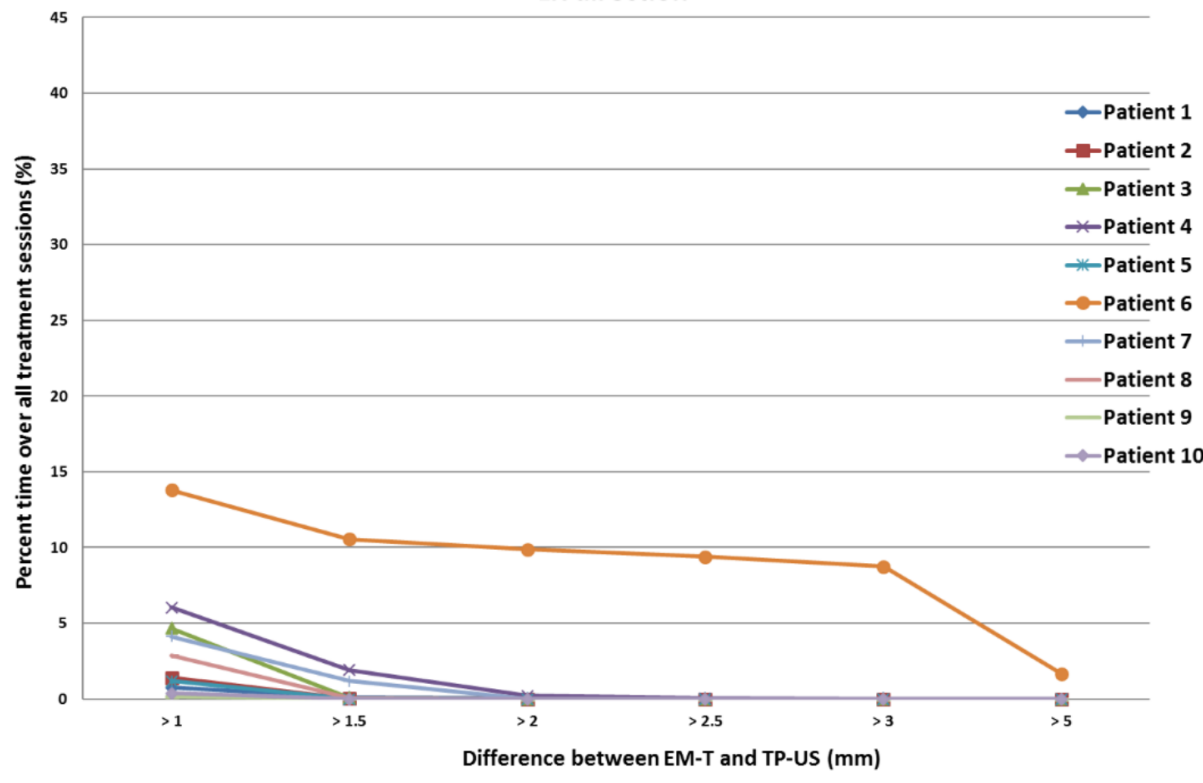

AP direction
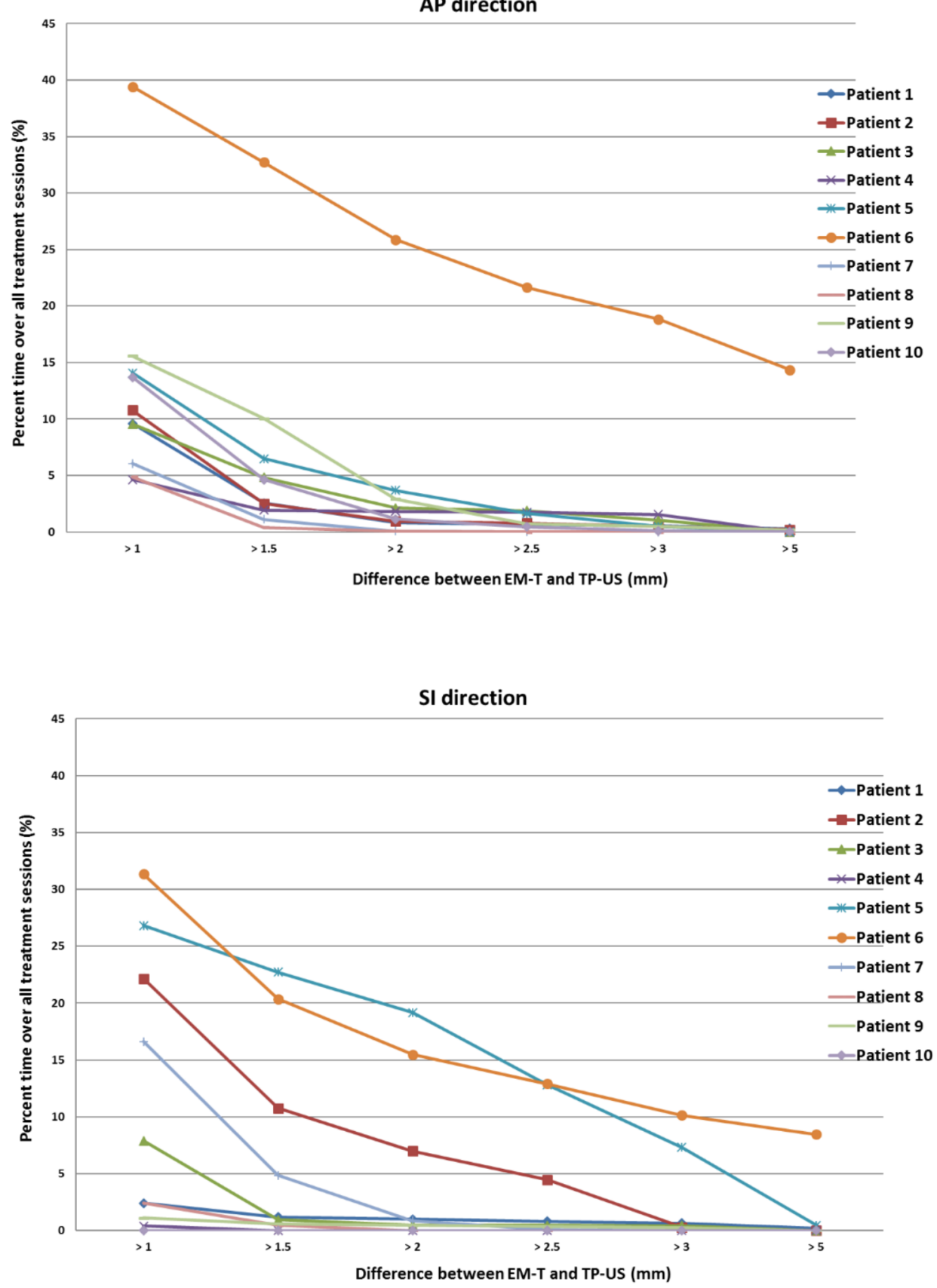

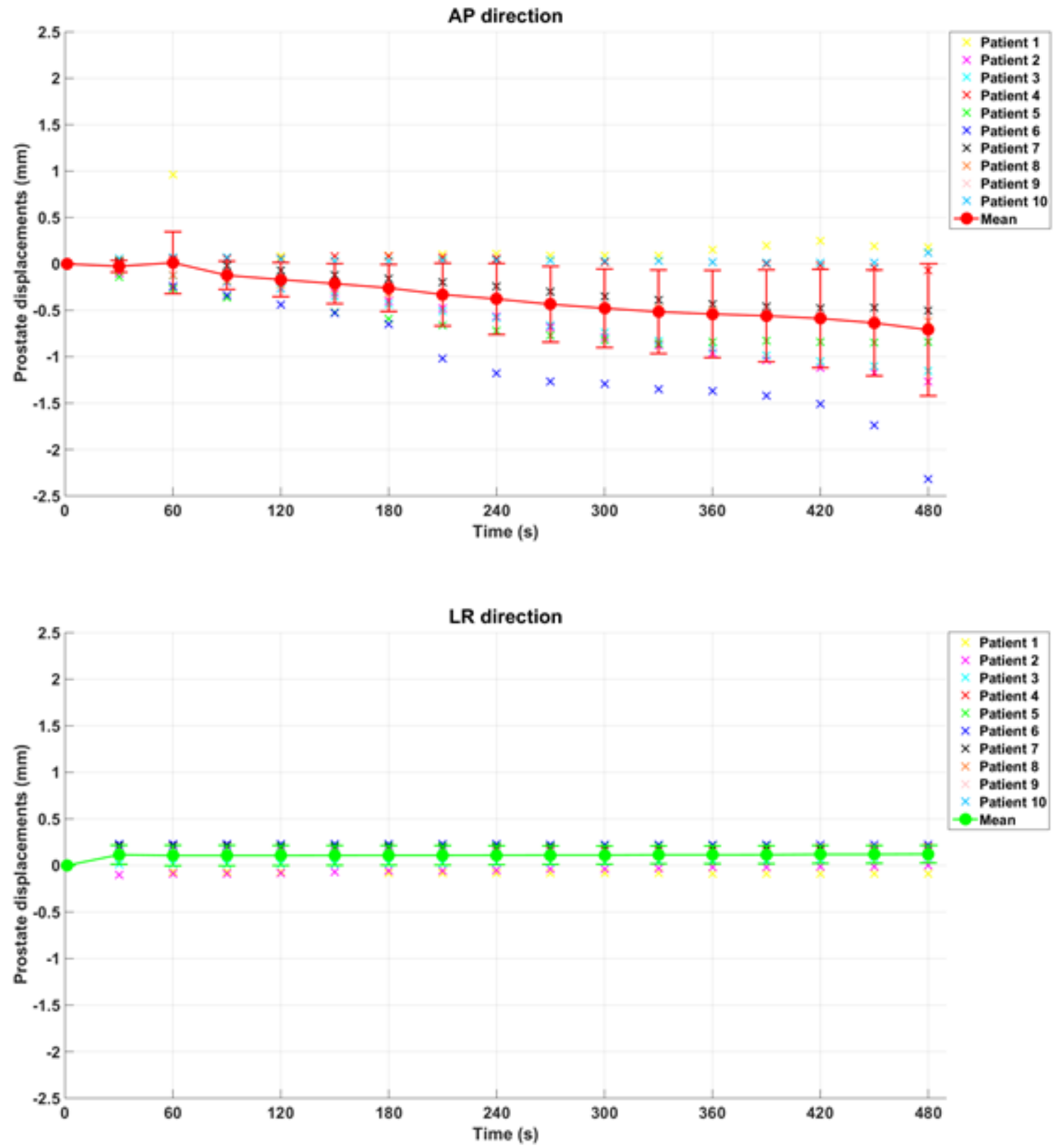

Patient 1

Patient 2

Patient 3

Patient 4

Patient 5

$\times$ Patient 6

$\times$ Patient 7

Patient 8

Patient 9

Patient 10

Moan

SI direction

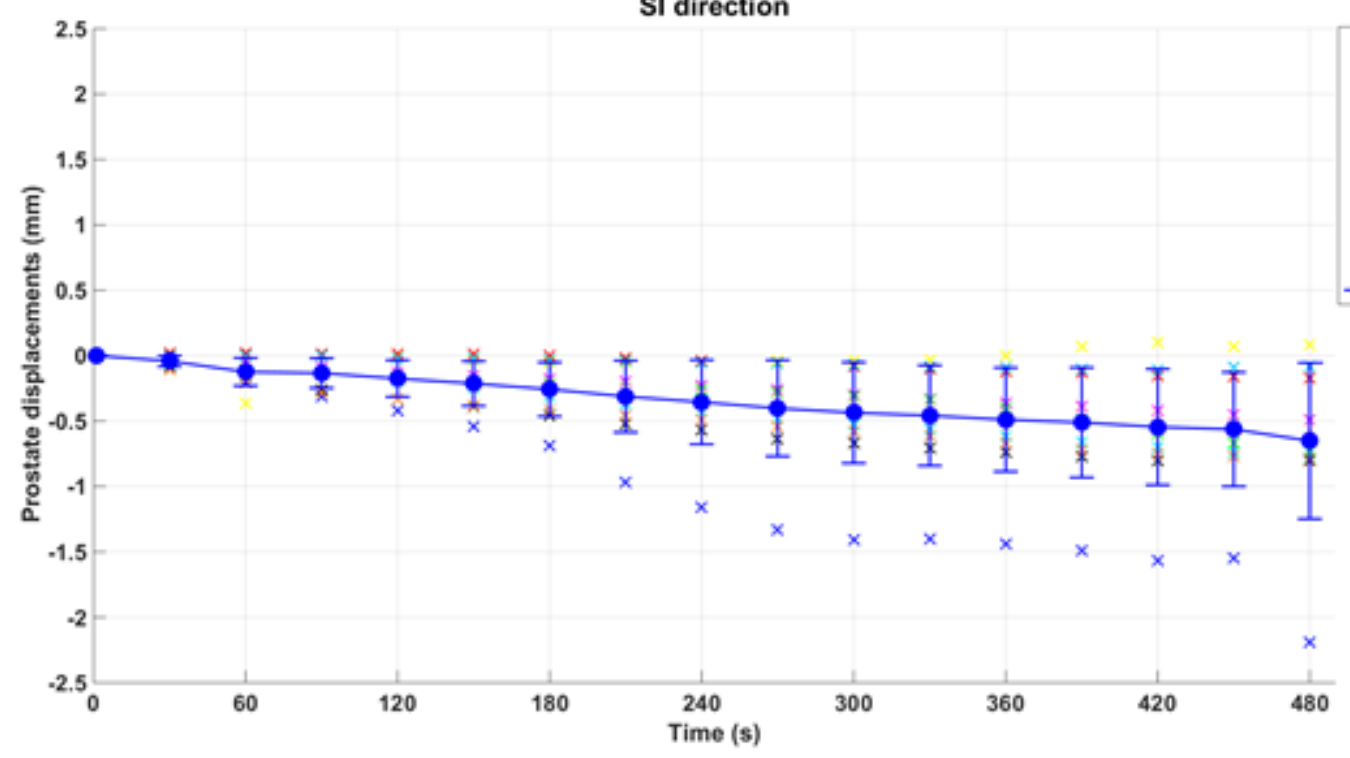

Patient 2

Patient 3

Patient 4

Patient 5

$\times$ Patient 6

$\times$ Patient 7

Patient 8

Patient 9

Patient 10 
A.
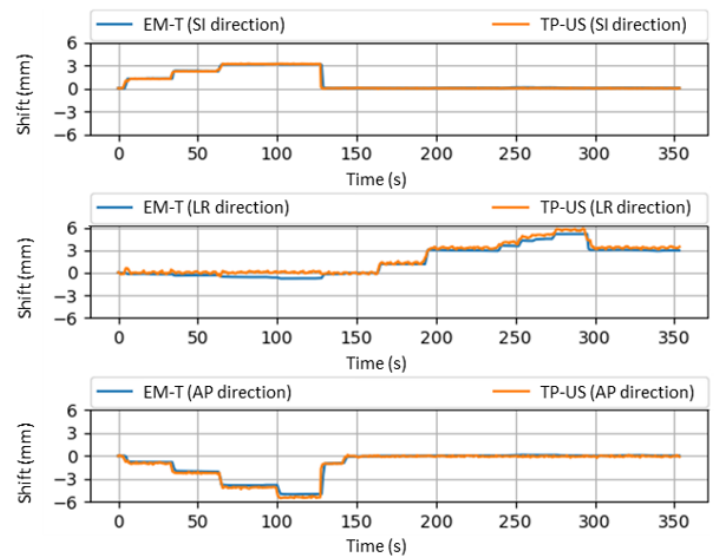

c.
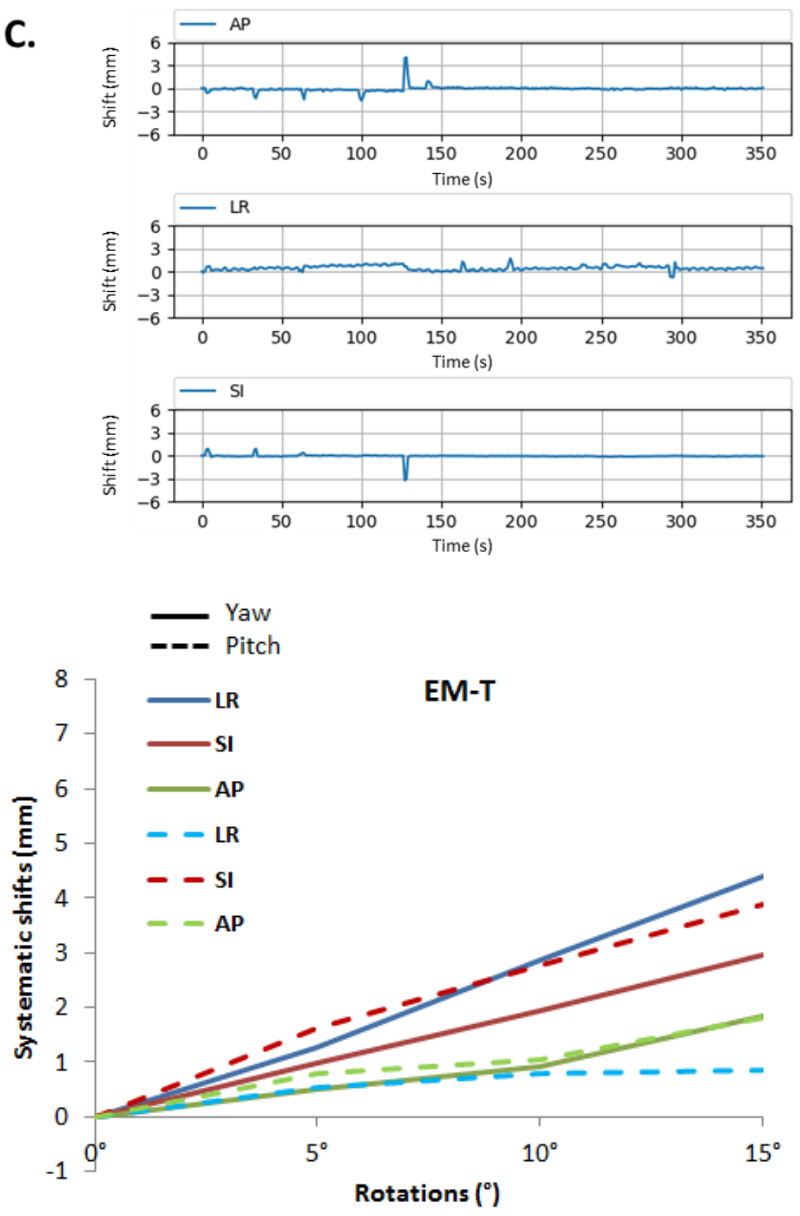

B.
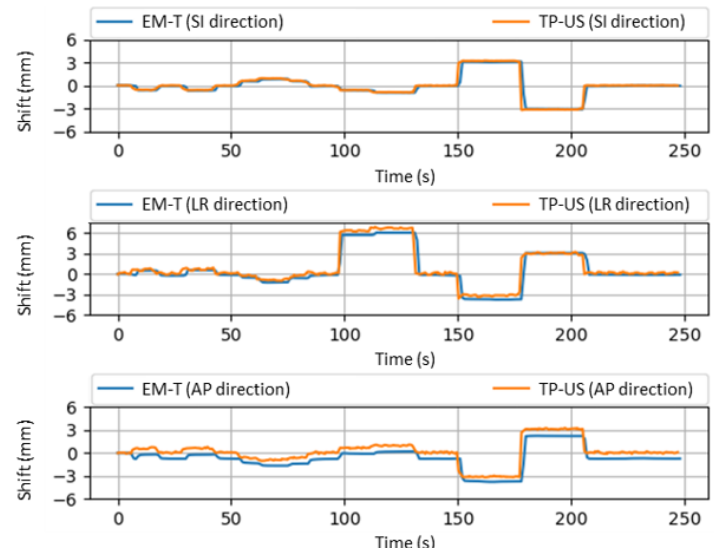

D.
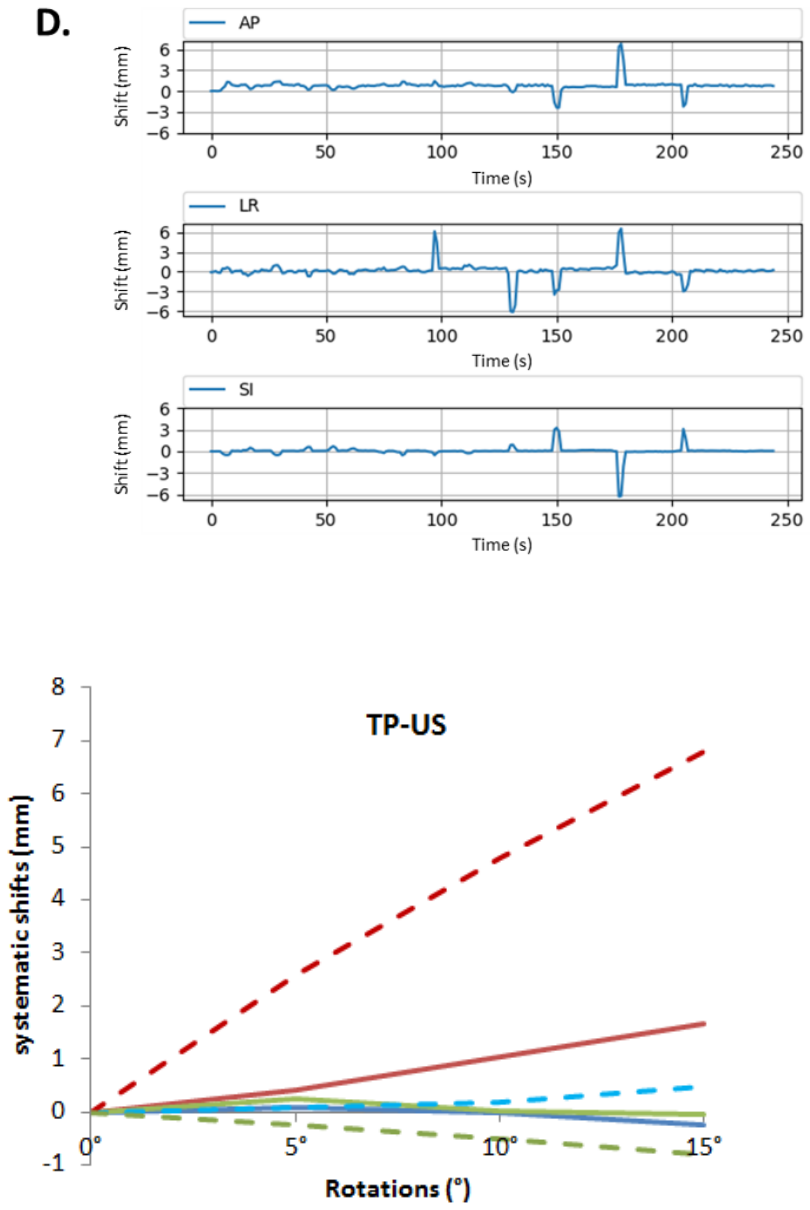


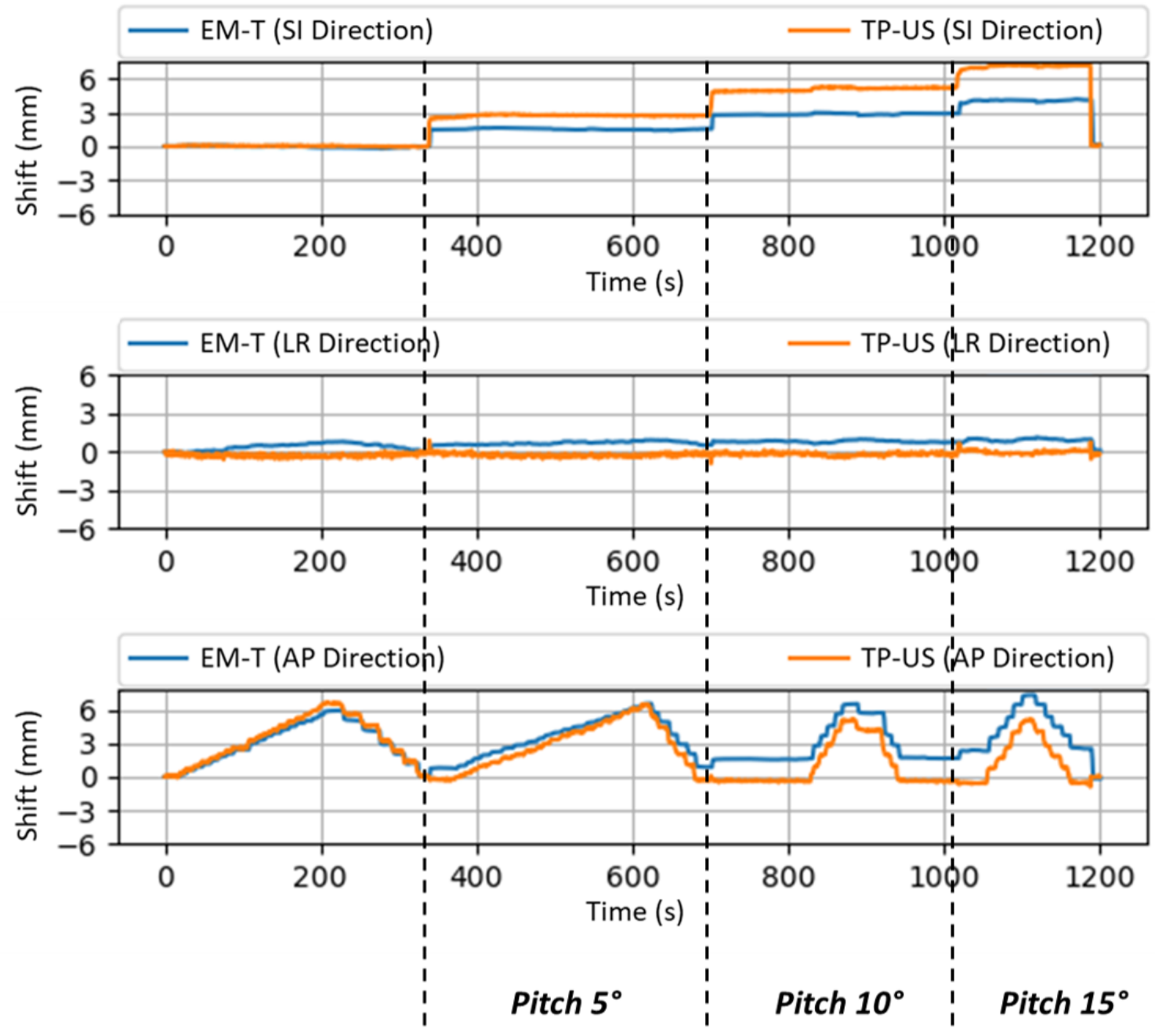

